Moderated Poster

11:45 PM - 1:05 PM

Wednesday, April 13, 2016

\title{
MP1 CORRECTING ATHEROGENIC EFFECTS OF LUPUS PLASMA ON MACROPHAGES WITH RESVERATROL AND MYCOPHENOLATE
}

NM Siegart, ${ }^{1,2}$ I Voloshyna, ${ }^{1}$ J DeLeon, ${ }^{2}$ SE Carsons, ${ }^{2,1}$ | Teboul, ${ }^{1,2}$ LJ Kasselman, ${ }^{1,2}$ J Mattana, ${ }^{2}$ AB Reiss ${ }^{1,2}$. 'Winthrop Research Institute, Winthrop-University Hospital, Mineola, NY, United States; ${ }^{2}$ Medicine, Winthrop-University Hospital, Mineola, NY, United States

10.1136/jim-2016-000080.13

Purpose of Study Premature atherosclerosis with coronary artery disease is a major cause of morbidity in Systemic Lupus Erythematosus (SLE). SLE patient plasma induces a pro-atherogenic profile of cholesterol transport genes in macrophages. A common immunosuppressive treatment for SLE, mycophenolate (MMF) reduces scavenger receptors thus reducing lipid influx. We have demonstrated atheroprotective properties of the polyphenol resveratrol on cholesterol efflux. This study determines whether MMF and resveratrol work synergistically to regulate cholesterol transport in macrophages exposed to pro-atherogenic SLE plasma.

Methods Used THP-1 human macrophages $\left(10^{6} / \mathrm{ml}\right)$ were incubated in 10\% SLE plasma with: media (control); MMF $(1 \mu \mathrm{g} / \mathrm{ml})$; resveratrol $(50 \mu \mathrm{M})$; and $\mathrm{MMF}+$ resveratrol. After $24 \mathrm{~h}$ incubation, total RNA and protein were isolated. Message level of scavenger receptors CD36, LOX1, and SRA1; and efflux proteins 27-hydroxylase, ATP binding cassette transporter (ABC)A1, and ABCG1 were evaluated by QRT-PCR and confirmed by immunoblot. Cholesterol efflux was measured by Amplex Red Cholesterol Assay kit run \pm cholesterol esterase.

Summary of Results In 10\% SLE plasma, MMF suppressed efflux genes ABCA1 and ABCG1 $(58.38 \pm 3.5 \%$ and $72.98 \pm 3.3 \%)$ vs. SLE plasma alone $(\mathrm{p}<0.0001)$ while $\mathrm{MMF}+$ resveratrol corrected this suppression. In SLE plasma, MMF+resveratrol decreased ScrA1 and LOX-1 by $15 \pm 2.5 \%$ and $47 \pm 1.0 \%$, respectively vs. resveratrol alone $(p<0.0001)$. SLE plasma promoted cholesterol accumulation in THP-1 macrophages and prevented efflux into medium. It increased the ratio of cholesterol esters to free cholesterol (ChE/FC). Resveratrol decreased intracellular cholesterol and restored $\mathrm{ChE} / \mathrm{FC}$ ratios to that of cells in healthy control plasma.

Conclusions MMF and resveratrol exhibit complimentary effects on macrophages exposed to SLE plasma. Both agents combined restore cholesterol influx and efflux gene expression to that of cells treated with control plasma. 
Resveratrol additionally reverses cholesterol accumulation caused by SLE plasma. Further evaluation of resveratrol $+\mathrm{MMF}$ in atherosclerosis in SLE may lead to improved treatment. 